\title{
Tendências genéticas para escores visuais de conformação, precocidade e musculatura à desmama de bovinos Nelore
}

\author{
Selma Forni ${ }^{1}$, Juliana de Freitas Federici ${ }^{2}$, Lucia Galvão de Albuquerque ${ }^{3,4}$ \\ ${ }^{1}$ Doutorado em Zootecnia, FCAV/UNESP - Jaboticabal. \\ 2 Zootecnista. \\ ${ }^{3}$ Departamento de Zootecnia, FCAV/UNESP - Jaboticabal, Pesquisadora do CNPq. \\ 4 Correspôndencias: Faculdade de Ciências Agrárias e Vaterinárias de Jaboticabal - Departamento de Zootecnia, Via de Acesso Prof. Paulo \\ Donato Castellane s/n, CEP: 14884-900, Jaboticabal - SP.
}

RESUMO - Os objetivos neste estudo foram estimar os coeficientes de herdabilidade e a mudança genética nos escores visuais de conformação (C), precocidade (P) e musculatura (M) à desmama de bovinos Nelore e avaliar as mudanças promovidas pelo programa de seleção ao qual os animais foram submetidos. Foram utilizados dados de 56.076 animais nascidos entre 1990 e 2002. Os componentes de variância foram estimados por máxima verossimilhança restrita e os valores genéticos foram preditos pelo método dos modelos mistos aplicando-se um modelo animal. As tendências genéticas foram estimadas pela regressão dos valores genéticos sobre o ano de nascimento dos animais. Os coeficientes de herdabilidade do efeito direto estimados foram de $0,122 \pm 0,012 ; 0,153 \pm 0,012$; e $0,124 \pm 0,012$ e, para o efeito materno, de $0,043 \pm 0,010$; 0,038 $\pm 0,009$; e $0,056 \pm 0,010$ para C, P e M, respectivamente. As tendências genéticas diretas estimadas foram de 0,013 ; 0,022 e 0,018 pontos de escore ao ano para $\mathrm{C}, \mathrm{P}$ e $\mathrm{M}$, respectivamente, o que representa incremento anual de 0,$42 ; 0,67$ e $0,60 \%$ ao ano em relação à média fenotípica. As tendências genéticas do efeito materno foram de $-0,0020 ;-0,0010$; e $-0,0009$ pontos de escore ao ano para $\mathrm{C}, \mathrm{P}$ e $\mathrm{M}$, respectivamente, o que representa uma mudança anual de $-0,07$; $-0,03$; e $-0,03 \%$ na média fenotípica da população.

Palavras-chave: crescimento, gado de corte, herdabilidade

\section{Genetic trends in Nelore cattle selected for visual scores of conformation, precocity and musculature at weaning}

\begin{abstract}
The aims of this study were to estimate heritability coefficients and genetic trends for visual scores of conformation (C), finishing precocity (P) and musculature (M) in Nelore beef cattle. Variance components and heritability coefficients were estimated by restrict maximum likelihood and breeding values were predicted by an animal mixed model using data from 56,076 animals born between 1990 and 2002. Genetic trends for each trait were estimated by the regression of breeding values on animal birth year. Heritability estimates for direct effect of C, P and M were respectively $0.122 \pm 0.012$, $0.153 \pm 0.012$ and $0.124 \pm 0.012$. In the same order of traits, maternal effect heritability estimates were respectively $0.043 \pm 0.010,0.038 \pm 0.009$ and $0.056 \pm 0.010$. Direct genetic trend estimates were respectively $0.013,0.022$ and 0.018 score units per year for $\mathrm{C}, \mathrm{P}$ and $\mathrm{M}$, which represent $0.42,0.67$, and $0.60 \%$ of the phenotypic average. Maternal effect genetic trends were respectively $-0.0020,-0.0010$ and -0.0009 for $\mathrm{C}, \mathrm{P}$ and $\mathrm{M}$, which represent $-0.07,-0.03$, and $-0.03 \%$ of the phenotypic average of the population.
\end{abstract}

Key Words: beef cattle, growth, heritability

\section{Introdução}

As características de crescimento dos animais, como o peso corporal, são importantes para a eficiência de qualquer sistema de produção de gado de corte e, por isso, têm sido utilizadas como critérios de seleção em diversos programas de melhoramento genético. Entretanto, as medidas de peso corporal em determinadas idades não são suficientes para avaliação do rendimento e da qualidade da carcaça do animal após o abate. Long (1973), ao propor o Sistema de Avaliação Ankony, afirmou que um programa de melhoramento genético embasado unicamente no ganho de peso não seria adequado, pois a composição do peso (proporção de massa muscular, gordura e ossos) não poderia ser ignorada, tendo em vista as demandas por carcaças de composição diferenciada no mercado.

Visando à avaliação indireta de características relacionadas à precocidade de terminação e à composição de 
carcaça, têm-se estudado algumas propostas de avaliação visual dos animais, entre elas, a utilização das características de conformação $(\mathrm{C})$, precocidade (P) e musculatura (M), que, segundo Jorge Jr. et al. (2001), procuram estimar o quanto o animal produziria de carne se fosse abatido no momento da avaliação, a capacidade do animal em acumular a quantidade de gordura mínima necessária para o abate com peso vivo não elevado e a quantidade de massas musculares presentes principalmente nos quartos traseiros, lombo, paleta e antebraço.

Resultados de pesquisas sugerem que a seleção baseada nos escores de $\mathrm{C}, \mathrm{P}$ e $\mathrm{M}$ poderia promover mudanças genéticas efetivas em rebanhos de gado de corte, visto que as estimativas de herdabilidade obtidas para essas características apresentaram magnitude média e suas correlações genéticas estimadas com os pesos em diferentes idades e a idade ao primeiro parto foram favoráveis (Cardoso et al., 1998; Koury Filho, 2001; Jorge Jr., 2002). Entretanto, as mudanças genéticas promovidas por um programa de melhoramento em uma população devem ser avaliadas periodicamente quanto à sua magnitude e direção. As estimativas das tendências genéticas das características selecionadas em determinado período após o início do processo de seleção é uma forma de se avaliar o progresso genético de um rebanho e decidir sobre ações futuras.

Os objetivos neste trabalho foram estimar os parâmetros e as tendências genéticas dos escores visuais de conformação, precocidade e musculatura à desmama em um rebanho Nelore e avaliar as mudanças promovidas pelo programa de seleção ao qual os animais foram submetidos.

\section{Material e Métodos}

Os dados utilizados neste trabalho foram obtidos em uma empresa agropecuária que se dedica à bovinocultura de corte, localizada no estado de São Paulo. O sistema de produção na empresa se baseia na utilização de pastagens tropicais. Foram disponibilizados 56.076 registros de C, P e $\mathrm{M}$, medidos à desmama, de animais Nelore, machos e fêmeas, nascidos entre os anos 1990 e 2002.

No programa de melhoramento genético em que participa o rebanho, os animais são avaliados e selecionados à desmama ( \pm 6 meses) e ao sobreano ( \pm 17 meses). Os valores genéticos das características $\mathrm{C}, \mathrm{P}$ e $\mathrm{M}$ à desmama represen$\operatorname{tam} 8,16$ e $16 \%$, respectivamente, do índice desmama e 4, 8 e $8 \%$ do índice final, enquanto os valores genéticos de $\mathrm{C}$, $\mathrm{P}$ e M ao sobreano representam 4, 8 e 8\%, respectivamente, do índice final. Esses escores são atribuídos aos animais no momento da pesagem e as notas variam de 1 a 5 , de modo que cada animal é avaliado relativamente ao seu grupo de manejo. A nota de cada animal para cada característica é definida de acordo com a observação do desempenho médio do grupo e depende da concordância entre três avaliadores. Em cada período de avaliação, 10\% das fêmeas e 50\% dos machos são descartados. Assim, aproximadamente $81 \%$ das fêmeas e $25 \%$ dos machos nascidos anualmente permanecem no rebanho ou são comercializados como reprodutores.

Foram desconsiderados os animais desmamados com menos de 140 dias e mais de 259 dias de idade. Os grupos contemporâneos foram definidos pela junção das variáveis: fazenda de nascimento e de desmama, grupo de manejo de nascimento e de desmama, sexo, ano e estação de nascimento (verão ou inverno). Grupos contemporâneos com menos de seis animais foram desconsiderados, sendo avaliados 56.076 registros de animais distribuídos em 867 grupos contemporâneos.

Os escores de C, P e M foram pré-ajustados para os efeitos de data juliana de nascimento, idade da vaca (mãe do animal avaliado) e idade do animal à desmama utilizando-se os fatores de correção estimados por Jorge Jr. et al. (2004), que estudaram detalhadamente a variação dos escores visuais em função destes efeitos ambientais. Esses autores modelaram esses efeitos comparando diferentes modelos e empregando polinômios segmentados. O efeito da idade da vaca foi modelado por um polinômio segmentado com dois segmentos quadráticos com nó aos sete anos, a data juliana de nascimento, por um polinômio segmentado com três segmentos quadráticos com nó aos 185 e aos 280 dias, e a idade à desmama, por um polinômio ordinário quadrático. Os fatores de correção foram estimados considerando os efeitos genéticos direto e materno utilizando-se um grande conjunto de dados da raça Nelore do qual fazem parte os registros utilizados neste trabalho.

Os componentes de variância foram estimados por Máxima-Verossimilhança Restrita utilizando-se o método não-derivativo descrito por Smith \& Graser (1986) no programa computacional MTDFREML (Boldman et al., 1993). Foram empregados modelos animais unicaracterística que incluíram os efeitos aleatórios de ambiente permanente materno e genético materno, visto que a inclusão destes efeitos aumentou significativamente o logaritmo da função de máxima-verossimilhança, segundo o teste de razão de verossimilhança (Hogg \& Craig, 1995). A forma matricial desses modelos pode ser descrita por:

$$
y=X b+Z_{1} a+Z_{2} m+W c+e
$$

em que $y=$ vetor de dados (C, P ou $\mathrm{M})$ ajustados para os efeitos de idade da vaca ao parto, data juliana de nasci- 
mento e idade do bezerro à desmama; $X=$ matriz de incidência para o efeito fixo grupo contemporâneo; $b=$ vetor do efeito fixo grupo contemporâneo; $Z_{l}=$ matriz de incidência para o efeito genético aditivo direto; $a=$ vetor de valores genéticos aditivos diretos dos animais; $Z_{2}=$ matriz de incidência para o efeito genético aditivo materno; $m=$ vetor de valores genéticos aditivos maternos; $W=$ matriz de incidência para o efeito de ambiente permanente da mãe; $c=$ vetor dos efeitos de ambiente permanente da mãe; $e=$ resíduo.

As estimativas de tendências genéticas para as características $\mathrm{C}, \mathrm{Pe} \mathrm{M}$ foram obtidas pela regressão dos valores genéticos sobre o ano de nascimento dos animais, por meio do procedimento "reg" do programa computacional SAS (1998). Em virtude do pequeno número de animais avaliados nascidos em outros anos, foram considerados 55.410 animais nascidos no período de 1991 a 2001 para estimação das tendências genéticas.

\section{Resultados e Discussão}

As médias e os desvios-padrão das características idade da vaca, data juliana de nascimento e idade do bezerro à desmama, bem como os efeitos de meio para os quais os escores de $\mathrm{C}, \mathrm{P}$ e $\mathrm{M}$ foram pré-ajustados, podem ser observados na Tabela 1.

Tabela 1 - Médias e desvios-padrão das características idade da vaca (anos), idade do bezerro à desmama (dias) e data juliana de nascimento

Table 1 - Means and standard deviations of age of dam (years), weaning age (days) of calf and Julian birth date

\begin{tabular}{lcc}
\hline $\begin{array}{l}\text { Característica } \\
\text { Trait }\end{array}$ & $\begin{array}{c}\text { Média } \\
\text { Mean }\end{array}$ & $\begin{array}{c}\text { Desvio-padrão } \\
\text { Standard deviation }\end{array}$ \\
\hline $\begin{array}{l}\text { Idade da vaca } \\
\text { Age of dam }\end{array}$ & 5,43 & 2,85 \\
$\begin{array}{l}\text { Idade do bezerro à desmama } \\
\begin{array}{l}\text { Weaning age of calf } \\
\text { Data juliana de nascimento } \\
\text { Julian birth date }\end{array}\end{array}$ & 188,17 & 20,22 \\
\hline
\end{tabular}

Tabela 2 - Médias, desvios-padrão e medianas das características conformação, precocidade e musculatura medidas à desmama

Table 2 - Means, standard deviations and medians of conformation, precocity and musculature at weaning

\begin{tabular}{lcc}
\hline Característica & $\begin{array}{c}\text { Média } \pm \mathrm{DP} \\
\text { Mean } \pm S D\end{array}$ & $\begin{array}{c}\text { Mediana } \\
\text { Median }\end{array}$ \\
\hline Crait & $3,08 \pm 0,85$ & 3 \\
Conformação (Conformation) & $3,26 \pm 0,84$ & 3 \\
Precocidade (Precocity) & $3,06 \pm 0,87$ & 3 \\
Musculatura (Musculature) & \\
\hline
\end{tabular}

Os valores médios e os desvios-padrão dos escores visuais de $\mathrm{C}, \mathrm{P}$ e $\mathrm{M}$ observados no rebanho são apresentados na Tabela 2. Estas médias são semelhantes às descritas por Jorge Jr. (2002), que, trabalhando com animais Nelore, relatou valores de $3,16 \pm 0,94 ; 3,19 \pm 0,96$ e 3,07 $\pm 0,97$ para C, $\mathrm{P} \mathrm{e} \mathrm{M}$, respectivamente. Cardoso et al. (2001) registraram valores médios inferiores e maiores desvios-padrão $(2,92 \pm 1,10 ; 3,09 \pm 1,08 ; \mathrm{e} 2,97 \pm 1,06$ unidades de escore para $\mathrm{C}, \mathrm{P}$ e M) em um rebanho Angus.

As estimativas dos componentes de variância e os coeficientes de herdabilidade para as três características podem ser observados na Tabela 3. Os coeficientes de herdabilidade direta estimados para os escores de $\mathrm{C}, \mathrm{Pe} \mathrm{M}$ foram semelhantes aos encontrados por Cardoso et al. (1998), que analisaram dados coletados à desmama em um rebanho Santa Gertrudes e relataram estimativas de herdabilidade de 0,10 para $\mathrm{C}, 0,15$ para $\mathrm{P}$ e 0,11 para $\mathrm{M}$. Koury Filho (2001), avaliando dados de animais Nelore, estimou coeficientes de herdabilidade semelhantes, variando entre 0,11 e 0,13 para $\mathrm{C}$, entre 0,09 e 0,11 para $\mathrm{P}$, e entre 0,16 e 0,18 para $\mathrm{M}$.

As estimativas obtidas neste estudo foram inferiores aos resultados encontrados por Eler et al. (1996), que avaliaram dados de outros rebanhos Nelore e estimaram valores de herdabilidade entre 0,20 e 0,23 para os escores de $\mathrm{C}, \mathrm{P}$ e $\mathrm{M}$. Valores ainda maiores foram descritos por Jorge Jr. (2002), que estimou herdabilidade de 0,33 para $C$; 0,39 para $\mathrm{P}$ e 0,34 para $\mathrm{M}$ na mesma raça. Em função das estimativas de herdabilidade encontradas neste e em outros rebanhos, espera-se que as características avaliadas respondam positivamente à seleção, entretanto, em períodos curtos respostas de pequena magnitude devem ser esperadas. A seleção direta para os escores visuais de $\mathrm{C}, \mathrm{P}$ e $\mathrm{M}$ deve apresentar pequenas taxas de ganho anual.

Os coeficientes de herdabilidade estimados para o efeito materno foram de pequena magnitude. Eler et al. (1996), também estudando animais da raça Nelore, relataram estimativas de herdabilidade maiores para os efeitos maternos das características C e P $(0,11$ e 0,08 , respectivamente) e menor estimativa para a herdabilidade materna de $\mathrm{M}(0,04)$. Entretanto, Jorge Jr. (2002) relatou estimativas menores para o efeito materno $(0,01,0,01$ e 0,03 , para $\mathrm{C}, \mathrm{P}, \mathrm{e} \mathrm{M}$, respectivamente). Assim, espera-se que não haja mudanças significativas nos escores visuais de $\mathrm{C}, \mathrm{P}$ e $\mathrm{M}$ decorrentes do efeito genético materno quando praticada seleção.

Os coeficientes de regressão dos valores genéticos aditivos direto e materno para os escores de $\mathrm{C}, \mathrm{P}$ e $\mathrm{M}$ sobre o ano de nascimento dos animais estão apresentados na Tabela 4. Os coeficientes de determinação dos modelos $\left(\mathrm{R}^{2}\right)$ foram todos superiores a 0,90 , evidenciando que o modelo 
Tabela 3 - Estimativas das variâncias genética direta $\left(\sigma^{2}{ }_{a}\right)$, genética materna $\left(\sigma_{m}^{2}\right)$, de ambiente permanente $\left(\sigma^{2}{ }_{c}\right)$ e residual $\left(\sigma^{2}{ }_{e}\right)$, herdabilidades direta $\left(h^{2}\right)$ e materna $\left(h^{2}{ }_{m}\right)$ e proporção da variância fenotípica decorrente do efeito de ambiente permanente $\left(c^{2}\right)$, dos escores visuais de conformação $(C)$, precocidade $(P)$ e musculatura $(M)$

Table 3 - Estimates of genetic direct $\left(\sigma_{a}^{2}{ }_{a}\right)$, genetic maternal $\left(\sigma_{m}^{2}\right)$, permanent environmental $\left(\sigma^{2}{ }_{c}\right)$ and residual $\left(\sigma^{2}{ }_{e}\right)$ variances, direct $\left(h^{2}{ }_{d}\right)$ and maternal $\left(h^{2}{ }_{m}\right)$ heritability and proportion of phenotypic variance due to permanent environmental effect $\left(c^{2}\right)$ for conformation $(C)$, precocity $(P)$ and musculature (M) visual scores

\begin{tabular}{lcccccc}
\hline & $\sigma_{\mathrm{a}}^{2}$ & $\sigma_{\mathrm{m}}^{2}$ & $\sigma_{\mathrm{c}}^{2}$ & $\sigma_{\mathrm{e}}^{2}$ & $\begin{array}{c}\mathrm{h}^{2}{ }_{\mathrm{d}} \pm \text { erro-padrão } \\
h^{2}{ }_{d} \pm \text { standard desviation }\end{array}$ & $\begin{array}{c}\mathrm{h}^{2}{ }_{\mathrm{m}} \pm \text { erro-padrão } \\
h^{2}{ }_{m} \pm \text { standard desviation }\end{array}$ \\
\hline $\mathrm{C}$ & 0,088 & 0,031 & 0,109 & 0,491 & $0,122 \pm 0,012$ & $0,043 \pm 0,010$ \\
$\mathrm{P}$ & 0,111 & 0,028 & 0,113 & 0,472 & $0,153 \pm 0,012$ & $0,038 \pm 0,009$ \\
$\mathrm{M}$ & 0,108 & 0,043 & 0,123 & 0,485 & $0,124 \pm 0,012$ & $0,056 \pm 0,010$ \\
\hline
\end{tabular}

Tabela 4 - Tendência genética anual (unidade/ano) para os efeitos direto e materno, de machos e fêmeas, dos escores de conformação $(C)$, precocidade $(P)$ e musculatura $(M)$

Table 4 - Annual genetic trend (unity/year) of direct and maternal effects, of males and females, for conformation (C), precocity $(P)$ and musculature (M) visual scores

\begin{tabular}{|c|c|c|c|c|c|c|}
\hline & \multicolumn{2}{|c|}{$\mathrm{C}$} & \multicolumn{2}{|c|}{$\mathrm{P}$} & \multicolumn{2}{|c|}{ M } \\
\hline & $\begin{array}{c}\text { Direto } \\
\text { Direct }\end{array}$ & $\begin{array}{l}\text { Materno } \\
\text { Maternal }\end{array}$ & $\begin{array}{c}\text { Direto } \\
\text { Direct }\end{array}$ & $\begin{array}{l}\text { Materno } \\
\text { Maternal }\end{array}$ & $\begin{array}{l}\text { Direto } \\
\text { Direct }\end{array}$ & $\begin{array}{l}\text { Materno } \\
\text { Maternal }\end{array}$ \\
\hline b_total (b_total) & 0,013 & $-0,002$ & 0,022 & $-0,001$ & 0,018 & $-0,0009$ \\
\hline$\%$ da média fenotípica (\% of phenotypic mean) & 0,42 & $-0,07$ & 0,67 & $-0,03$ & 0,60 & $-0,03$ \\
\hline
\end{tabular}

$\mathrm{b}=$ coeficientes de regressão dos valores genéticos dos animais sobre os respectivos anos de nascimento.

$b=$ regression coefficients of breeding values on animal birth year.

linear foi adequado para explicar a evolução dos valores genéticos diretos e maternos, em função do ano de nascimento, para as três características avaliadas.

Para a característica $\mathrm{C}$, foi observado um ganho genético de menor magnitude, o que era esperado, pois, nos índices utilizados para a seleção, a ponderação atribuída a $\mathrm{C}$ foi menor que aquelas atribuídas às outras características avaliadas. Espera-se que ganhos genéticos de maior magnitude possam ser observados com a utilização de um índice de seleção que contenha os pesos econômicos das diferentes características, estimados a partir dos dados de custos e receitas do próprio rebanho, visto que ganhos genéticos relevantes foram observados mediante seleção baseada em um índice em que os valores genéticos para as diferentes características são ponderados de forma empírica.

As retas de regressão dos valores genéticos direto e materno sobre a ano de nascimento dos animais para os escores de C, P e M podem ser observadas na Figura 1. Não foram encontradas na literatura estimativas de tendências genéticas para os escores visuais de C, P e M que servissem como base para comparações. Entretanto, os incrementos anuais representados como porcentagem da média fenotípica encontrados neste trabalho mantiveram-se na amplitude de valores descritos na literatura para características de crescimento (Euclides Filho et al., 2000; Fernandes et al., 2002; Garcia et al., 2003). Mello et al. (2002) estimaram tendências genéticas diretas de 0,66 e $0,75 \%$ de ganho anual na média fenotípica para as características peso à desmama e peso aos 12 meses, respectivamente, em um rebanho Canchim. Ferraz Filho et al. (2002) relataram ganhos inferiores, de $0,08 \%$ de aumento, na média fenotípica do peso aos 205 dias de animais Tabapuã. Mucari \& Oliveira (2003) também relataram pequenos ganhos genéticos anuais em características de crescimento de um rebanho Guzerá: $0,10 \%$ de aumento da média fenotípica no peso aos oito meses de idade; $0,02 \%$ no peso aos 12 meses de idade; $0,06 \%$ no peso aos 18 meses de idade; e $0,14 \%$ no peso aos 24 meses de idade.

Os valores estimados para a tendência genética do efeito materno foram negativos e muito próximos de zero, indicando que a mudança genética decorrente desse efeito nas três características foi praticamente nula. Em poucas pesquisas foram avaliadas as mudanças promovidas pelo efeito genético materno em populações bovinas. Analisando o peso à desmama em um rebanho Tabapuã, Ferraz Filho et al. (2002) também relataram valores praticamente nulos para tendência genética materna, $0,01 \%$ de aumento na média fenotípica anual do peso à desmama. Valores inexpressivos também foram relatados por Euclides Filho et al. (2000) e Mello et al. (2002), ao avaliarem a tendência genética materna do peso à desmama em outras raças. Espera-se que a contribuição do efeito genético materno para mudanças nos escores visuais avaliados seja muito pequena, haja vista a pequena fração da variância fenotípica atribuída a este efeito. 

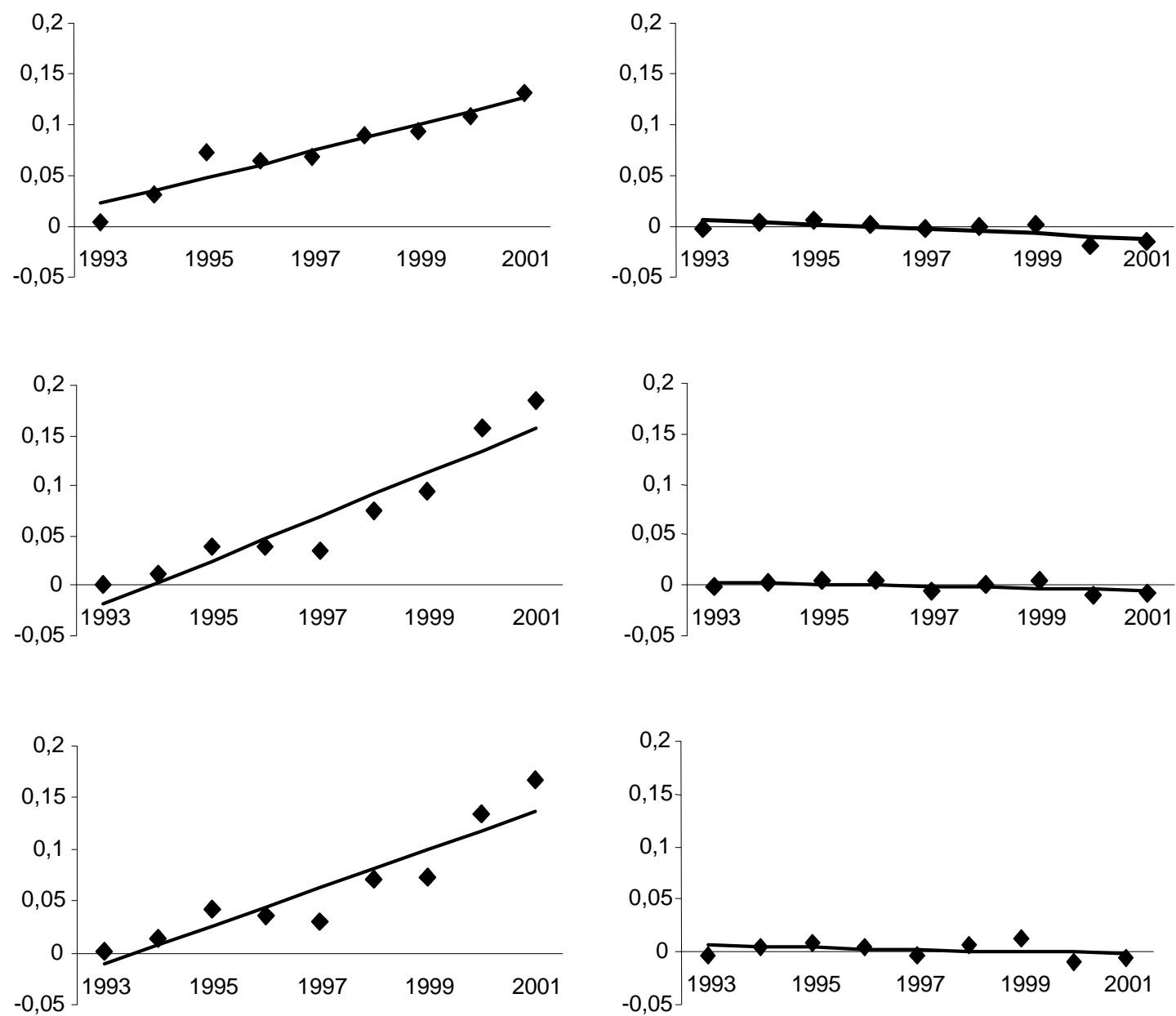

Figura 1 - Tendências genéticas aditiva direta (à esquerda) e materna (à direita) para os escores de conformação, precocidade e musculatura (de cima para baixo, respectivamente).

Figure 1 - Additive direct (left side) and maternal (right side) genetic trends for conformation, precocity and musculature visual scores (top-down direction, respectively).

\section{Conclusões}

As características conformação, precocidade e musculatura sofrem grande influência do ambiente. Assim, respostas à seleção direta de pequenas magnitudes devem ser esperadas e a predição de méritos genéticos acurados depende da avaliação de progênies numerosas.

As tendências genéticas alcançadas para os escores visuais sugerem que os critérios de seleção utilizados no rebanho estudado resultaram em progresso genético e que o ganho genético materno pouco influenciou o ganho genético total do rebanho.

\section{Literatura Citada}

BOLDMAN, K.; KRIESE, L.; Van VLECK, L.D. A manual for use of MTDFREML: a set of programs to obtain estimates of variances and covariances (DRAFT). Lincoln: Department of Agriculture, Agriculture Research Service, 1993. 120p.
CARDOSO, F.F.; CARDELLINO, R.A.; CAMPOS, L.T. Parâmetros genéticos para escores de avaliação visual à desmama em bovinos da raça Santa Gertrudes. In: REUNIÃO ANUAL DA SOCIEDADE BRASILEIRA DE ZOOTECNIA, 35.,1998, Botucatu. Anais... Botucatu: Sociedade Brasileira de Zootecnia, 1998. p.506.

CARDOSO, F.F.; CARDELliNO, R.A.; CAMPOS, L.T. Fatores ambientais sobre escores de avaliação visual à desmama em bezerros Angus criados no Rio Grande do Sul. Revista Brasileira de Zootecnia, v.30, p.318-325, 2001.

ELER, J.P.; FERRAZ, J.B.S.; SILVA, P.R. Parâmetros genéticos para peso, avaliação visual e circunferência escrotal na raça Nelore, estimados por modelo animal. Arquivo Brasileiro de Medicina Veterinária e Zootecnia, v.48, p.203-213, 1996.

EUCLIDES FILHO, K.; SILVA, L.O.C.; ALVES, R.G.O. et al. Tendência genética na raça Gir. Pesquisa Agropecuária Brasileira, v.35, p.787-791, 2000.

FERNANDES, H.D.; FERREIRA, G.B.B.; RORATO, P.R.N. Tendências e parâmetros genéticos para características prédesmama em bovinos da raça Charolês criados no Rio Grande do Sul. Revista Brasileira de Zootecnia, v.31, p.321-330, 2002.

FERRAZ FILHO, P.B.; RAMOS, A.A.; SILVA, L.O.C. et al. Tendência genética dos efeitos direto e materno sobre os pesos à desmama e pós-desmama de bovinos da raça Tabapuã no Brasil. Revista Brasileira de Zootecnia, v.31, p.635-640, 2002. 
GARCIA, F.Q.; FERRAZ FILHO, P.B.; SOUZA, J.C. et al. Tendências dos efeitos genéticos diretos e maternos do peso a desmama de bovinos da raça Nelore Mocha na região pecuária Campo Grande e Dourados - Mato Grosso do Sul. Archives of Veterinary Science, v.8, p.93-97, 2003.

HOGG, R.V.; CRAIG, A.T. Introduction to mathematical statistics. 5.ed. New Jersey: Prentice Hall, 1995. 564p.

JORGE JR., J.; DIAS, L.T.; ALBUQUERQUE, L.G. Fatores de correção de escores visuais de conformação, precocidade e musculatura, à desmama, para idade da vaca ao parto, data juliana de nascimento e idade à desmama em bovinos da raça Nelore. Revista Brasileira de Zootecnia, v.33, p.20442053, 2004.

JORGE JR., J. Efeitos genéticos e de ambiente sobre os escores visuais de conformação, precocidade e musculatura, no período pré-desmama, em bovinos da raça Nelore. Jabioticabal: Universidade Estadual Paulista, 2002. 76p. Dissertação (Mestrado em Zootecnia) - Universidade Estadual Paulista, 2002.

JORGE JR., J.; PITA, F.V.C.; FRIES, L.A. et al. Influência de alguns fatores de ambiente sobre os escores de conformação, precocidade e musculatura à desmama em um rebanho da raça Nelore. Revista Brasileira de Zootecnia, v.30, p.1697-1703, 2001 .

KOURY FILHO, W. Análise genética de escores de avaliações visuais e suas respectivas relações com desempenho ponderal na raça Nelore. Pirassununga: Universidade Estadual Paulista, 2001. 79p. Dissertação (Mestrado em Zootecnia) Universidade Estadual Paulista, 2001.
LONG, R.A. El sistema de evaluación de Ankony y su aplicación en la mejora del ganado. Colorado: Ankony Corporation, 1973. $21 \mathrm{p}$.

MELLO, S.P.; ALENCAR, M.M.; SILVA, L.O.C. et al. Estimativas de (co)variancias e tendências genéticas para pesos em um rebanho Canchim. Revista Brasileira de Zootecnia, v.31, p.1707-1744, 2002.

MUCARI, T.B.; OLIVEIRA, J.A. Análise genético-quantitativa de pesos aos 8, 12, 18 e 24 meses de idade em um rebanho da raça Guzerá. Revista Brasileira de Zootecnia, v.32, p.1604-1613, 2003.

STATISTICAL ANALYSIS SYSTEM - SAS. SAS/STAT user's guide. Version 6.12. 4.ed. Cary: 1998. 842p.

SMITH, S.P.; GRASER, H.U. Estimating variance components in a class of mixed models by restricted maximum likelihood. Journal of Dairy Science, v.69, p.1156-1165, 1986. 